ReiDoCrea

\title{
MOTIVACIÓN Y RENDIMIENTO ACADÉMICO: ¿CUMPLE SUS OBJETIVOS EL PLAN BOLONIA?
}

\section{MOTIVATION AND ACADEMIC ACHIEVEMENT: DOES BOLOGNA PLAN FULFILL ITS OBJECTIVES?}

Laura Fernandez García, José Antonio Mancebo Quirante, María Pilar Jiménez Álvarez, Teresa Ardanaz Sánchez, Noelia Fernández Sánchez y Alba María Montano de la Hoz. Universidad de Granada

\section{RESUMEN}

Diversos estudios han establecido que existe una relación directa entre la motivación y el rendimiento académico. La confluencia de estos dos factores resulta relevante en la formación del alumnado universitario, por esta razón, se decidió implantar en 2010 el Plan Bolonia, cuyo fin es aumentar la eficacia y calidad de la enseñanza, sustituyendo al Plan 2000. Este estudio tiene como objetivo, comprobar si estas mejoras son realmente eficaces, suponiendo un avance con respecto al plan 2000. Para esta investigación, se ha seleccionado a 103 estudiantes universitarios elegidos al azar, de ambos planes de estudios. Los resultados obtenidos han desmitificado los objetivos del Plan Bolonia,obteniendo una media de 5.01 en Motivación frente al 5.08 de Motivación del Plan 2000. Esto conlleva a que el Rendimiento académico sea más elevado en el Plan 2000, consiguiendo una media de 7.16 respecto al 6.71 del Plan Bolonia.

Palabras clave: Plan de estudios, Motivación, Rendimiento académico.

\begin{abstract}
Several studies have established that exists a direct relationship between Motivation and Academic Achievement. The confluence of these two factors is eminent in the university students' education. For this reason, in 2010, the Bologna Plan is deployed as purpose to increase the efficiency and the quality of education, by replacing Plan 2000 . This study aims to verify if those improvements are really effectives, assuming an advance with regard to Plan 2000. For this investigation, 103 university students have been selected at random, of both curriculums. The results obtained have demystified the Bologna Plan's objectives, obtaining an average of 5.01 in Motivation against 5.08 of Plan 2000's Motivation. All of these mean that Academic achievement is higher in Plan 2000, getting an average of 7.16 , in respect of 6.71 of Bologna Plan's Academic achievement.
\end{abstract}

Key words: Curriculum, Motivation, Academic achievement.

\section{INTRODUCCIÓN}

Está demostrado que existen dos aspectos importantes en la vida académica y que estos son la motivación y el rendimiento académico. Ambos conceptos están incluidos en la escuela, colegio, instituto y universidad. En el siguiente estudio nos centraremos en la relación entre la motivación y el rendimiento académico, concretamente en el ambiente 
universitario. Nuestra intención es conocer, en primer lugar, si existe relación entre estos dos conceptos; y después saber qué plan de estudios de los dos actuales que existen en el sistema universitario de España es más efectivo y favorable teniendo en cuenta estas dos variables, en concreto el Plan 2000, incluyendo licenciaturas y diplomaturas, y el Plan Bolonia dedicado sólo a estudiantes de grado.

Empezaremos explicando el rendimiento académico, que es un indicador de eficacia y calidad educativa. Las variables que intervienen en él son: factores contextuales, donde se incluyen variables socioculturales (clima educativo y familiar, origen sociocultural), institucionales (tipo y tamaño del centro) y pedagógicas (expectativas y actitudes del profesor). $\mathrm{Y}$ hay que tener también en cuenta factores personales, dentro de los que se incluyen variables demográficas (sexo, edad), cognitivas (aptitudes intelectuales y motivación) y actitudinales (satisfacción, autoconcepto); Todos estos factores son, a su vez, los principales indicadores de riesgo de fracaso académico.

En relación a la diversidad de variables vinculadas con un rendimiento académico eficaz, hemos orientado nuestra investigación hacia el estudio de la relación existente entre éste y la motivación. Según Kleinginna y Kleinginnna (1981), la motivación es una fuerza interna que mueve al organismo para llevar a cabo un determinado comportamiento. El término motivación dentro de la psicología ha sido empleado para definir el inicio, la dirección, la intensidad y la persistencia de una conducta (Geen, 1995). Los componentes de la motivación son cuatro: activación (fuerza o energía que justifica que un comportamiento llegue a producirse), persistencia (se asume que el grado de ésta es un índice del nivel de motivación de un individuo ante una situación), intensidad (a mayor intensidad mayor motivación), y dirección (mostrar preferencia por una alternativa frente a otra).

Diversos estudios han afirmado correlaciones positivas entre estas dos variables, entre ellos figura el trabajo de Celorrio (1999), Hidalgo (1987) y García Llamas (1985). Hidalgo (1987) afirma que la motivación general, la motivación específica y el rendimiento académico están asociados significativamente. Por otro lado, Llamas encontró que los motivos de elección de una carrera son un predictor significativo del rendimiento académico.

A partir de estas dos variables se han elaborado distintos planes de estudio. Uno de ellos es el Plan 2000 que se encuentra en proceso de extinción, y que busca la especialización en los distintos ámbitos de conocimiento con el fin de conseguir una preparación para asumir las exigencias del mercado laboral. Está dividido en dos ciclos; el primero más dirigido a la teoría y los conocimientos generales y el segundo, hacia la profundización y puesta en práctica de los conocimientos adquiridos. Además de los créditos troncales y optativos, el estudiante debe de obtener créditos de libre configuración obtenidos en materias que pueden elegirse en la propia facultad o en centros especializados. La carga lectiva oscila entre 20 y 30 horas semanales siendo el total del curso entre 60 y 90 créditos por año académico.

Más nuevo e innovador es el Plan Bolonia, elaborado para una mayor eficacia del alumnado y para mejorar con respecto a los planes anteriores. Busca la diversificación curricular permitiendo que la universidad aproveche su capacidad de innovación, sus fortalezas y oportunidades. También incluye créditos europeos (ECTS) como unidad de 
medida que refleja los resultados del aprendizaje y volumen de trabajo realizado por el estudiante para alcanzar los objetivos establecidos en el plan de estudio, poniendo en valor la motivación y el esfuerzo del estudiante para aprender.

Los objetivos planteados que pretendemos abordar en este estudio son: comprobar si existe correlación entre la motivación y el rendimiento académico orientada a la formación universitaria y también conocer si las bases de mejora que se proponen conseguir con el Plan Bolonia se han alcanzado.

A continuación se presentan las hipótesis que vamos a utilizar como base para esta investigación:

Hipótesis 1: Una mayor motivación en los estudios universitarios provoca un mayor rendimiento académico.

Hipótesis 2: Existe una mayor motivación y un mayor rendimiento académico en Grado que en el Plan 2000.

\section{MÉTODO}

\section{Participantes}

La muestra está formada por estudiantes universitarios entre 18 y 47 años, siendo representativa de ambos sexos. La edad media es de 21.8 años ( $S D=2.86)$. Todos ellos estudian diferentes titulaciones en universidades públicas españolas con distinta ubicación geográfica, por lo que su nivel educativo es medio-alto.

La muestra total se compone de 103 sujetos seleccionados al azar. A partir de esta muestra, se han establecido dos grupos en función de su pertenencia al Plan 2000 (más comúnmente denominado Licenciatura) o al Sistema de Transferencia de Créditos Europeos o Grado. Según esta variable, la muestra se compone de 50 participantes pertenecientes a Licenciatura y 53 a Grado.

\section{Instrumentos}

Para la obtención de los datos necesarios se construyó un cuestionario que incluía las siguientes medidas:

- Datos socio-demográficos: edad, sexo, estudios y facultad, si trabajaba además de estudiar y si realizaba un Grado o una Licenciatura.

- Cuestionario de Estrategias de Aprendizaje y Motivos de Estudio (CEAM) (Roces, Tourón, González-Torres, 1995), del cual se han utilizado los ítems correspondientes a la evaluación de la motivación (parte A del cuestionario, 31 ítems). Los sujetos indicaban su respuesta en una escala Likert con 7 opciones de respuesta, siendo el 1 "No me describe en absoluto" y el 7 "Me describe totalmente". 
- Rendimiento académico que se ha evaluado utilizando la nota media del expediente académico (sobre 10) hasta la fecha en que desarrolló el estudio. Se empleó esta medida ya que es la que mejor refleja en forma numérica esta variable.

Este cuestionario se ha administrado en formato papel y vía online, a partir de la herramienta de creación de encuestas de Google.

Los materiales utilizados fueron un ordenador, en caso de ser necesaria la consulta del expediente académico o como forma de cumplimentar el cuestionario y bolígrafos para rellenar los cuestionarios. Para el análisis estadístico de los datos obtenidos se utilizó el programa SPSS 20.0 de IBM.

\section{Procedimiento}

El experimento se inició con la administración del cuestionario a diferentes universitarios de España mediante una encuesta online o pasándolo con cuestionarios en papel. El motivo de hacerlo online fue el de captar a la mayor cantidad posible de sujetos de diferentes universidades y facultades, no solo de Granada. En todo momento se aseguró el anonimato a los participantes.

\section{ANÁLISIS DE LOS RESULTADOS}

Los resultados se presentan en primer lugar haciendo referencia a la relación entre la motivación y el rendimiento académico; y en segundo lugar se comprueba si existe mayor motivación y rendimiento académico en Grado que en el Plan 2000.

\section{- Motivación y rendimiento académico}

Los resultados obtenidos en el análisis de los datos en cuanto a la motivación, señalan una media $=156,35(S D=18.15)$; y en el rendimiento académico se ha obtenido una media correspondiente a $6.80(S D=1.32)$.

Para analizar si existe correlación entre motivación y rendimiento académico se ha utilizado la correlación de Pearson, y teniendo en cuenta que $(p<.05)$ se ha obtenido una correlación significativa, $r=.23$.

\section{TABLA 1. RELACIÓN ENTRE MOTIVACIÓN Y RENDIMIENTO ACADÉMICO}

\begin{tabular}{llll}
\hline Media & $\begin{array}{l}\text { Desviación } \\
\text { típica }\end{array}$ & $\begin{array}{l}\text { Coeficiente de } \\
\text { correlación de } \\
\text { Pearson }\end{array}$ & $\begin{array}{l}\text { Significación } \\
\text { Pilal }\end{array}$ \\
\hline
\end{tabular}

\begin{tabular}{lll}
\hline Motivación & 153.35 & 18.15
\end{tabular}

$\begin{array}{lll}\text { Rendimiento académico } \quad 6.804 & 1.32\end{array}$ 


\section{- Mayor motivación y rendimiento académico en Grado que en el Plan 2000}

Para llevar a cabo este análisis de resultado se han realizado las medias y desviaciones típicas de ambos planes de estudio y las correlaciones existentes entre motivación y rendimiento académico de cada plan, utilizando la correlación de Pearson, teniendo en cuenta que $(p<.05)$.

En primer lugar en Grado se han obtenido las siguientes medias y desviaciones típicas: en motivación $(M=5.01 ; S D=.55) ; y$ en rendimiento académico $(M=6.71 ; S D=.96)$. En cuanto a si existe correlación entre motivación y rendimiento académico en Grado, el resultado obtenido es de .065 por tanto, no es significativa.

En cuanto al Plan 2000 se han obtenido las siguientes medias y desviaciones típicas: en motivación $(M=5.08 ; S D=.63)$; y en rendimiento académico $(M=7,16 ; S D=.72)$, sin embargo sí existe correlación significativa entre motivación y rendimiento académico en estos estudiantes, siendo de .318 .

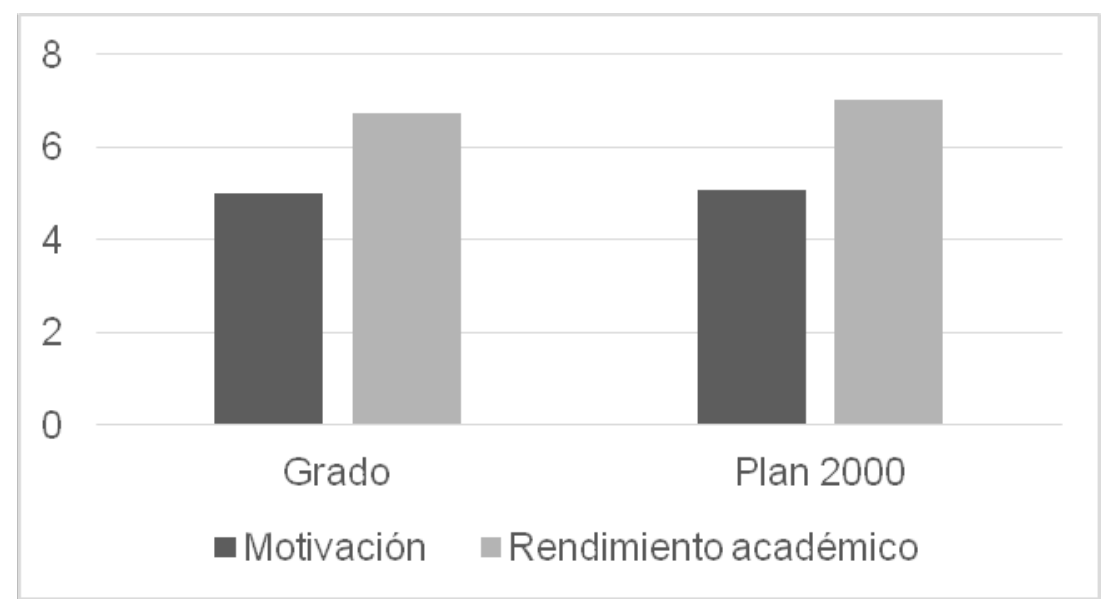

TABLA 2. RESULTADOS DE DIFERENCIACIÓN ENTRE GRADO Y LICENCIATURA

\begin{tabular}{llcccc}
\hline & Media & $\begin{array}{l}\text { Desviación } \\
\text { típica }\end{array}$ & $\begin{array}{l}\text { Coeficiente } \\
\text { de } \\
\text { correlación } \\
\text { de Pearson }\end{array}$ & $\begin{array}{l}\text { Significación } \\
\text { bilateral }\end{array}$ \\
\hline Grado & Motivación & 5.01 & .55 & .065 & .642 \\
& $\begin{array}{l}\text { Rendimiento } \\
\text { académico }\end{array}$ & 6.71 & .96 & & $.318^{*}$ \\
2000 & $\begin{array}{l}\text { Motivación } \\
\text { Plan }\end{array}$ & 5.08 & .63 & & .024 \\
& $\begin{array}{l}\text { Rendimiento } \\
\text { académico }\end{array}$ & 7.16 & .73 & & \\
\hline
\end{tabular}




\section{DISCUSIÓN}

La hipótesis 1, una mayor motivación en los estudios universitarios provoca un mayor rendimiento académico, se cumple. Es decir, existe una correlación positiva, por tanto a mayor motivación mayor rendimiento académico. Esto se puede deber a que cuando un estudiante comienza una carrera universitaria es porque está motivado a tener un futuro mejor con lo que estudia o bien porque adquiere un conocimiento sobre un área concreta sobre la que le gusta aprender. Esta motivación lleva al alumno a prepararse e interesarse más por lo que aprende y sacar buenas notas en los exámenes lo que repercute en su rendimiento académico.

Por otra parte, la hipótesis 2, existe una mayor motivación y un mayor rendimiento académico en Grado que en el Plan 2000, no se cumple ya que existe una puntuación significativa en cuanto a motivación y rendimiento académico en los alumnos del plan 2000 que no se ha encontrado en los alumnos del plan Bolonia. Y en comparación entre las medias se observa un mayor rendimiento académico entre los estudiantes del Plan 2000 que en los de Grado. Una de las razones que pueden justificar este resultado es la urgencia de los estudiantes del plan 2000 a la hora de terminar sus estudios por la extinción de este plan. Esta situación puede dar lugar a que la motivación por aprobar las asignaturas sea mayor en estos estudiantes y esto afecte al rendimiento académico.

Otra razón puede ser que las premisas del Plan Bolonia no se lleven a cabo de una manera adecuada en la práctica y lo único que se haya hecho es seguir con lo anterior pero haciendo todas las asignaturas cuatrimestrales llevando a que lo único que se quiera sea aprobar para no examinarse en septiembre disminuyendo la motivación.

Por todo esto, podemos sacar como conclusión que el Plan 2000 es más efectivo que el moderno Plan Bolonia en lo referente a la motivación, y por ello se obtiene un mayor rendimiento académico. Esto nos lleva a cuestionarnos si las manifestaciones de muchos alumnos y profesores de las diferentes universidades en contra del Plan Bolonia podrían ser fundamentadas por este motivo: la falta de efectividad a la hora de conseguir que los alumnos estén motivados con el fin de obtener un mayor rendimiento académico.

Por último, cabe citar las limitaciones que presenta este estudio entre las que se encuentran el tamaño de la muestra, la motivación por contestar al cuestionario y el no poder controlar que hacían los sujetos mientras contestaban online.

Por otra parte hay que tener en cuenta que los sujetos pueden haber mentido al responder sobre su nota media de expediente y poner una más alta de la que se tiene en realidad debido a la deseabilidad social.

Para finalizar, otro factor importante son los años que llevan los estudiantes en la carrera ya que no es lo mismo un alumno de primero que tiene mucha ilusión por estudiar lo que le gusta que un repetidor o alguien de último curso que quiere terminar. Se podría crear otra línea de investigación sobre la motivación y el rendimiento académico en estos estudiantes, para ver las diferencias existentes entre ambos. 


\section{REFERENCIAS BIBLIOGRÁFICAS}

Artunduaga Murillo, M. (2008). Variables que influyen en el rendimiento académico en la universidad.

Recuperado 22 de abril de http://www.slideshare.net/1234509876/variablesdel-rendimiento-academico-universidad

Cazorla, M.C. Una aproximación a los aspectos positivos y negativos derivados de la puesta en marcha del Plan Bolonia en la Universidad española.

Recuperado el 18 de abril de http://eciencia.urjc.es/bitstream/10115/5764/1/PDF.pdf

Colmenares, M. y Delgado, F. (2008). La correlación entre rendimiento académico y motivación de logro: elementos para la discusión y reflexión. Revista electrónica de humanidades, educación y comunicación social (REDHECS), 3 (5), 179-191.

Edel, R. (2003). El rendimiento académico: concepto, investigación y desarrollo. Revista electrónica iberoamericana sobre la calidad, eficacia y cambio en educación (REICE), 1 (2), 1-15.

España. Real Decreto 1393/2007, de 29 de octubre, del Código educativo. Boletín Oficial del Estado, 30 de octubre de 2007, núm. 260, p. 44037.

España. Real Decreto 1497/1987, de 27 de noviembre, del Código educativo. Boletín oficial del Estado, 14 de diciembre de 1987, núm. 298, p. 36639.

García Bacete, F.J. y Doménech Betoret, F. (1997). Motivación, aprendizaje y rendimiento escolar. Revista electrónica de Motivación y Emoción, 1 (0). Universidad Jaume I de Castellón.

Recuperado el 16 de abril de 2013 de http://reme.uji.es/articulos/pa0001/texto.html

González, C. (2003). Factores determinantes del bajo rendimiento académico en educación secundaria. Tesis doctoral no publicada, Universidad Complutense de Madrid, Madrid, España.

Herrera, M.E., Nieto, S., Rodriguez, M.J. y Sánchez, M.C. (1999). Factores implicados en el rendimiento académico de los alumnos. Universidad de Salamanca. Revista de Investigación Educativa, 17(2), 413-421.

Núñez, J.C. (2009). Motivación, Aprendizaje y Rendimiento Académico.X Congreso Internacional Galego-Portugués de Psicopedagogía. Universidade do Minho.

Quirós Expósito, P. Cabestrero Alonso, R. (2008). Funciones activadoras: principios básicos de la motivación y la emoción. Editorial Universitaria Ramón Areces. 